\title{
Intravitreal injections during the COVID-19 pandemic era
}

\author{
Selda Celik Dulger ${ }^{1}$, Mehmet Citirik ${ }^{1}$, Esra Bahadir Camgoz ${ }^{1}$ and Mehmet Yasin Teke ${ }^{1}$ \\ ${ }^{1}$ Ophthalmology Department, University of Health Sciences, Ankara Ulucanlar Eye Training and Research Hospital, Ankara, \\ Turkey
}

\begin{abstract}
Background: This study aimed to evaluate the clinical characteristics and changes in the number of patients receiving intravitreal injections (IVIs) at a tertiary hospital during the coronavirus disease 2019 (COVID-19) pandemic as compared to the pre-pandemic period.

Methods: This retrospective, cross-sectional study included 3,211 patients with retinal disease, who received IVIs of anti-vascular endothelial growth factor (anti-VEGF) between January and May 2020. This 5-month period was divided into a pre-pandemic and a pandemic period. Clinical and demographic data were collected and were compared between the patients in each period. All COVID-19 infection precautions were implemented to minimize the potential transmission of COVID-19 to both healthcare workers and patients.

Results: A total of 3,211 IVIs were administered to patients with diabetic retinopathy, age-related macular degeneration, retinal vein occlusion, and other retinal conditions. Diabetic retinopathy was the most common indication for treatment in the pre-pandemic as well as pandemic periods. Bevacizumab (Avastin ${ }^{\circledR}$, Roche) was the most common IVI type, followed by aflibercept (Eylea ${ }^{\oplus}$, Bayer). Of 3,211 IVIs, 2,943 (91.7\%) were administered during the pre-pandemic period and 268 (8.3\%) during the pandemic period. There was a statistically significant decrease in injections between the pre-pandemic and pandemic periods, with an overall reduction of $90.8 \%$ in IVIs $(P<0.05)$. No cases of confirmed transmission of COVID-19 or complications associated with IVIs were recorded.

Conclusions: This study showed that the number of IVIs and patient visits decreased significantly, by more than 10-fold, during the lockdown period. These findings show that COVID-19 has turned the management of sight-threatening eye diseases into a challenging process and must be addressed if future healthcare restrictions are imposed.

\section{KEYWORDS}

COVID-19, retina, intravitreal injection, age-related macular degeneration, diabetic retinopathy, retinal vein occlusion, anti-vascular endothelial growth factor, anti-VEGF, bevacizumab, aflibercept
\end{abstract}

\section{INTRODUCTION}

Intravitreal injection (IVI) with anti-vascular endothelial growth factor agents (anti-VEGF) is accepted as a standard treatment for various retinal diseases, including diabetic retinopathy, age-related macular degeneration (AMD), and retinal vein occlusion [1]. These conditions can result in irreversible vision loss if treatment

Correspondence: Esra Bahadir Camgoz, University of Health Sciences, Ulucanlar Eye Training and Research Hospital. Ulucanlar Cd. No: 5906230 Altindag, Ankara, Turkey. E-mail: esrabhdr@gmail.com. ORCID iD: https://orcid.org/0000-0003-2962-4960

How to cite this article: Celik Dulger S, Citirik M, Bahadir Camgoz E, Teke MY. Intravitreal injections during the COVID-19 pandemic era. Med Hypothesis Discov Innov Optom.2021 Summer; 2(2): 50-55. https://doi.org/10.51329/mehdioptometry125

Received: 16 June 2021; Accepted: 05 November 2021

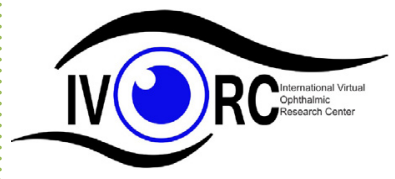

Copyright (C) Author(s). This is an open-access article distributed under the terms of the Creative Commons Attribution-NonCommercial 4.0 International License (http://creativecommons.org/licenses/by-nc/4.0/) which permits copy and redistribute the material just in noncommercial usages, provided the original work is properly cited. (c) (i) \$ 
is delayed. Moreover, most patients with retinal disease are older and generally have an underlying medical condition. Although the coronavirus disease 2019 (COVID-19) affects people of all ages, older people with underlying health conditions have a higher risk of developing severe symptoms [2, 3$]$.

The retina clinics are one of the places in which transmission of COVID-19 to both healthcare workers and patients must be minimized. Performing intravitreal injections (IVIs) is a particular issue faced in retinal clinics during this challenging period. Many guidances exist and several studies have been conducted on how often IVIs are performed and how patients should be prioritized during the pandemic period [4-9].

We aimed to evaluate the clinical characteristics and changes in the number of patients who received IVIs at our tertiary hospital during the COVID-19 and pre-pandemic periods. Furthermore, we described our strategy for and experience in preventing the transmission of the SARS-COV-2 virus to healthcare staff.

\section{METHODS}

This retrospective, cross-sectional study was conducted at a tertiary hospital (Ulucanlar Eye Training and Research Hospital, Ankara, Turkey) between January and May 2020. A total of 3,211 patients with retinal disease who received IVIs of anti-VEGF were included in the study. The Ethics Committee of Ankara Training and Research Hospital approved the study, and written consent was obtained from all patients. This study adhered to the tenets of the Declaration of Helsinki.

Injection data for all included cases, in addition to patient demographics, diagnosis, type of IVI, and medical history, were collected from patients' records. The 5 months' study period was divided into two groups: the prepandemic period and the pandemic period, based on before and after March 15, 2020, respectively. This date was chosen, as the first case of COVID-19 was diagnosed in our country on March 11, 2020, and anti-COVID-19 measures were implemented in our hospital as of March 15, 2020. Thus, the incidence rate of IVIs and the retinal diagnosis were compared to the duration of 2.5 months before and after March 15, 2020.

Statistical analyses were performed using IBM SPSS Statistics for Windows (version 21.0, IBM Corp., Armonk, NY, USA). Categorical variables are presented as frequencies and percentages. An independent $t$-test was used to compare differences between the groups, as the data were normally distributed. A $P$-value $<0.05$ was considered statistically significant.

\section{RESULTS}

This study included 3,211 patients who received an IVI, among whom 1,566 (48.8\%) were females and 1,645 (51.2\%) were males, with a mean \pm standard deviation (SD) age of $64.87 \pm 11.11$ years (range: 16-93 years). In the pre-pandemic period, diabetic retinopathy was the most common diagnosis, accounting for 1,608 (54.6\%) cases. Other cases involved AMD $(n=895,30.4 \%)$, retinal vein occlusion $(n=314,10.7 \%)$, and other retinal diseases $(n=126,4.3 \%)$, respectively. The order of diagnoses was the same during the COVID-19 pandemic period. There was a significant decline $(P<0.01)$ in the number of patients with diabetic retinopathy attending eye clinic visits during the pandemic period (pre-pandemic: $n=1608$; pandemic: $n=123$; change: $-92.3 \%$ ). Similarly, there was a significant decrease in the number of patients with AMD attending the clinic (prepandemic: $n=895$; pandemic: $n=90$; change: $-89.9 \%)(P<0.01)$. A similar rate of decrease was observed in patients with retinal vein occlusion (pre-pandemic: $n=314$; pandemic: $n=40,-87.2 \%)(P<0.01)$. Other retinal diseases, including vitreous hemorrhage, cystoid macular edema secondary to pathologies other than diabetic retinopathy, and choroidal neovascular membrane secondary to non-AMD diseases, also showed a significant decrease during this period (Table 1).

Bevacizumab (Avastin ${ }^{\oplus}$, Roche, Basel, Switzerland) was the most commonly used anti-VEGF agent in both periods, followed by aflibercept (Eylea ${ }^{\circledR}$ Bayer, Leverkussen, Germany), dexamethasone implant (Ozurdex ${ }^{\circledast}$,

Table 1. Diagnoses of patients receiving intravitreal injection during a pre-pandemic and a pandemic period

\begin{tabular}{|c|c|c|c|c|}
\hline Main disease, $\mathbf{n}(\%)$ & Pre-pandemic & Pandemic & Change & P-value \\
\hline Diabetic retinopathy & $1608(54.6)$ & $123(45.9)$ & $-92.3 \%$ & $<0.01$ \\
\hline AMD & $895(30.4)$ & $90(33.6)$ & $-89.9 \%$ & $<0.01$ \\
\hline Retinal vein occlusion & $314(10.7)$ & $40(14.9)$ & $-87.2 \%$ & $<0.01$ \\
\hline Others $^{*}$ & $126(4.3)$ & $15(5.6)$ & $-88.0 \%$ & $<0.01$ \\
\hline
\end{tabular}

Abbreviations: n, number; \%, percentage; AMD, Age-related macular degeneration. $P<0.05$ is shown in bold. Note: asterisk indicates other diseases included vitreous hemorrhage, cystoid macular edema secondary to reasons other than diabetic retinopathy, and choroid neovascular membrane secondary to non-AMD. 
Table 2. Characteristic of anti-VEGF intravitreal injections during a pre-pandemic and a pandemic period

\begin{tabular}{|c|c|c|c|c|c|}
\hline \multicolumn{2}{|l|}{ Variable } & Pre-pandemic & Pandemic & Change & $P$-value \\
\hline \multicolumn{2}{|c|}{ Total number of intravitreal injections, $\mathbf{n}(\%)$} & $2943(91.7)$ & $268(8.3)$ & $-90.8 \%$ & $<0.05$ \\
\hline \multirow[t]{5}{*}{ Drugs, n (\%) } & Bevacizumab (Avastin ${ }^{\circledR}$, Roche) & $1296(44)$ & $130(48.5)$ & $-89.9 \%$ & $<0.01$ \\
\hline & Aflibercept (Eylea ${ }^{\circledR}$, Bayer) & $806(27.4)$ & $82(30.6)$ & $-89.8 \%$ & $<0.01$ \\
\hline & Corticosteroid implant (Ozurdex®, Allergan) & $309(10.5)$ & $17(6.3)$ & $-94.4 \%$ & $<0.01$ \\
\hline & Ranibizumab (Lucentis®, Novartis) & $297(10.1)$ & $17(6.3)$ & $-94.2 \%$ & $<0.01$ \\
\hline & Triamcinolone & $235(8.0)$ & $22(8.3)$ & $-90.6 \%$ & $<0.01$ \\
\hline
\end{tabular}

Abbreviations: anti-VEGF, anti-vascular endothelial growth factor agents; $n$, number; $\%$, percentage. $P<0.05$ is shown in bold.

Allergan, Inc., Dublin, Ireland), and ranibizumab (Lucentis ${ }^{\circledast}$, Novartis, Basel, Switzerland) (Table 2). Of the 3,211 IVIs administered, 2,943 (91.7\%) were performed during the pre-pandemic period and $268(8.3 \%)$ were administered during the pandemic period. There was a significant decrease (more than 10 -fold) in the number of patients receiving an IVI during the pandemic period $(-90.8 \%, P<0.05)$ (Table 2). None of the patients receiving IVI developed endophthalmitis, retinal detachment, or other serious adverse effects. No cases of confirmed transmission of COVID-19 were recorded.

\section{DISCUSSION}

In this study, we found a marked reduction in the outpatient volume and IVIs administered in a retina clinic during the COVID-19 pandemic. Overall, we demonstrated that the COVID-19 pandemic significantly decreased both outpatient visits and intravitreal procedure volumes.

With the COVID-19 pandemic, the working strategies in the retina clinic of our hospital were markedly changed. Triage was performed on all ophthalmic patients, regardless of COVID-19 status, in line with the recommendations of the Turkish Ministry of Health [10]. All patients presenting for admission to the hospital had their temperature taken, and they were asked about having had contact with someone diagnosed with COVID-19 in the last 14 days, and having had a fever or cough in recent days. If the outcome of any of these inquiries indicated a risk of COVID-19, they were referred to an assigned hospital. Only patients without fever or contact history were allowed to enter our hospital. Eye-care providers applied universal personal protective measures, such as wearing surgical/N95 masks, gloves as necessity, protective clothing, face shields, and goggles [11]. They were educated about proper and frequent hand-washing and disinfection. Slit lamps were meticulously cleaned between use, and a transparent barrier was placed in the slit lamp device, between the ophthalmologist and the patient. During the examination and IVI, both the examiner and patient were required to wear a mask, and the room was ventilated frequently.

Transmission of COVID-19 is known to occur through respiratory droplets, aerosols, and direct/indirect contact. Because the safe distance to avoid droplet transmission is $1.5-2 \mathrm{~m}$, ophthalmology examination with close physical contact, unavoidable when using a slit lamp or ophthalmoscope, puts eye-care providers at greater risk for respiratory droplet transmission $[12,13]$. Overcrowding, inadequate ventilation, air conditioners, and large temperature differences in rooms increase the risk of COVID-19 infection $[14,15]$. In our clinic, all patients were encouraged to wear masks and were warned to pay attention to social distancing. The waiting room set-up was rearranged for 1.5-m social-distancing. No cases of confirmed transmission of COVID-19 were recorded.

IVIs have become the most commonly performed ophthalmic procedure [16]. On the one hand, patients are faced with many risk factors for the transmission of COVID-19 during ophthalmic examination and IVI. On the other hand, postponement of IVI can cause irreversible vision loss in some patients [17]. Prioritizing patients and treating those with the greatest medical need, while minimizing the risk of infection in both healthcare workers and patients seems to be the most important issue during the pandemic period [7-9]. In another study [18], triage criteria were implemented according to visual acuity, severity of the disease, and the status of the fellow eye. Treatment for patients with neovascular AMD or central retina vein occlusion (CRVO) in one eye, and with a visual acuity (VA) in the fellow eye below 20/60 Snellen, was considered to be highly urgent. Treatment for patients with bilateral neovascular AMD or bilateral CRVO and with a VA in both eyes above 20/60 Snellen, or patients with neovascular AMD in one eye and with a VA in the fellow eye between 20/60 and 20/40 Snellen, was considered urgent. For patients with neovascular AMD or CRVO and with a VA in the fellow eye $\geq 20 / 32$ Snellen; treatment was considered semiurgent. For patients with diabetic macular edema (DME), macular edema secondary to branch retinal vein occlusion, pseudophakic macular edema secondary to 
intraocular surgery, or AMD with a VA $<20 / 400$ in the affected eye, treatment was considered non-urgent [18].

Patients requiring IVIs in retina clinics have a particular risk of contracting COVID-19 [9]. Most of them are older and have an underlying systemic condition, such as diabetes mellitus, hypertension, chronic kidney disease, and respiratory disease [2]. Patients with comorbid hypertension, diabetes mellitus, and respiratory diseases were found to have a significantly higher prevalence of COVID-19 fatalities [3]. These patients are also at potential risk of irreversible vision loss if left untreated. It has been emphasized that patients with AMD (particularly in the first 2 years of treatment), neovascular glaucoma, new retinal vein occlusion, and new cases with severe vision loss must be prioritized and the treatment regimen must be maintained [18]. Postponement may not be a major problem for patients with DME and branch retinal vein occlusion (except for patients with severe vision loss due to recent DME and retinal vein occlusion) [19]. However, it is emphasized that prolonged postponement ( $>4-6$ months) should be avoided and rearrangement should be made within 2-3 months [19]. Our findings showed that COVID-19 turned the management of sight-threatening eye diseases into a challenging process. We found that the COVID-19 pandemic significantly decreased both outpatient visits and intravitreal procedure volumes.

A study involving 132 patients who received IVIs during the COVID-19 pandemic evaluated the mean period of delay, best-corrected visual acuity (BCVA), and central macular thickness. The mean delay was 6 weeks; only $20 \%$ of the patients were delayed for 8 weeks. Because of treatment delays, there was a significant decline in the mean BCVA and a significant increase in the mean central macular thickness on optical coherence tomography (OCT) [20]. Another study demonstrated a significant rise in vision-threatening complications, such as submacular hemorrhage, in patients with $\mathrm{AMD}$, because of increased non-attendance of clinic visits during the national COVID-19 lockdown [21]. We did not investigate the visual impact of delayed intravitreal anti-VEGF in the current study. However, the findings of those studies indicate that prioritizing treatment in such eyes should be considered, and the risks of sight-threatening consequences following prolonged treatment postponement need to be discussed thoroughly with patients.

The purpose of this study was to compare the frequency of retinal diagnoses and the number of IVIs performed between a pre-COVID-19 pandemic period (January 1 to March 15, 2020) and the first few months of the pandemic (March 15 to May 30, 2020). Almost half of our patients had diabetic retinopathy, about $30 \%$ of patients had AMD, and approximately $10 \%$ had retinal vein occlusion. Other diagnoses were vitreous hemorrhage, cystoid edema secondary to other conditions, and choroidal neovascularization secondary to non-AMD conditions. Although diabetic retinopathy represents the largest subgroup of patients, there was a significant decline in the number of patients with diabetic retinopathy attending the clinic during the pandemic period. Naravane et al. [22] reported that treatment delays had a negative impact on the visual and anatomic outcomes of patients with neovascular AMD and DME during the COVID-19 lockdown in Minnesota. They compared the VA and structural changes in the retina using ocular OCT and observed worse visual outcomes in the delayed treatment group [22]. However, in the current study, we only reported the frequency of cases between the pre-pandemic and pandemic period but have not yet investigated the visual outcomes.

Postponement of treatment in AMD may have more devastating effects on retinal morphology and may result in macular scarring or submacular hemorrhage. A previous study showed that treatment delay due to the COVID-19 pandemic caused the progression of neovascular AMD and visual impairment [23]. Similarly, in another study, more than half of neovascular AMD patients following a treat-and-extent protocol missed their scheduled IVI visits during the pandemic period, which resulted in a delay in their treatments. The delay of IVI treatment in these patients resulted in an increase in disease activity, as seen on OCT, and a decrease in BCVA [24]. The recommended treatment interval for patients with wet AMD is 8 weeks, and there is no clinical examination unless patients mention a significant decrease in vision [25]. For confirmed new wet AMD, clinical examination with a 3-IVI loading dose, followed by IVI at intervals of 8 weeks, with no clinic review is recommended [25]. Unfortunately, we found a notably decreased rate of IVI among patients with AMD. A similar decrease rate was observed in patients with retinal vein occlusion in the current study.

We hypothesize that the reduction in the number of patients with retinal disease may be due to the fear of patients with comorbidities, such as diabetes mellitus, hypertension, and older age, of becoming infected by going to the hospital. Statements by the government and healthcare professionals about avoiding hospitals, except for urgent situations, may be another factor. Postponement of appointments for non-urgent cases and social restrictions may be further reasons. In another study, 183 eyes of 144 patients who underwent IVI-based care from June 18, 2020, to August 7, 2020, were compared with 193 eyes of 154 patients who had IVI during the corresponding interval, 1 year earlier [26]. During the more recent period, 62 eyes of 46 patients were attended to later than scheduled, which represented a larger proportion of patients with delayed care as compared to the previous year. Return eyes with delayed treatment showed more decline in BCVA, poorer OCT results, 
which more frequently was switched to other IVI agents [26]. We found a significant decline in the number of patients with diabetic retinopathy, AMD, retinal vein occlusion, and other retinal diseases attending eye clinic visits during the pandemic period. However, the current study failed to investigate the visual impact of delayed adminstration of intravitreal anti-VEGF among those deemed to be at the risk of vision loss.

Importantly, our study demonstrated a significant reduction in the number of IVIs administered during a COVID-19 lockdown, with 2,943 injections provided in the pre-COVID-19 period, and 268 injections in the first 2.5 months of the COVID-19 pandemic period (decrease of 90.8\%). We found that bevacizumab (Avastin Roche) was the most common anti-VEGF type administered in both periods, due to economic reasons. There was a small, but not statistically significant, increase in the number of aflibercept (Eylea ${ }^{\oplus}$, Bayer) IVIs. The reason for this may be that aflibercept (Eylea ${ }^{\circledast}$, Bayer) is preferred by physicians because of its slightly longer potency [27].

Our results showed that a large percentage of patients were left untreated during a 2.5 -month period during the COVID-19 pandemic, and some of them probably developed irreversible vision loss. However, we realized that there was an increase in the number of patients attending retina clinics by the end of the restriction period. Yet, verifying this observation requires future research. The limitations of our study are that this was an analysis based on a single center and during a short pandemic study-period. Additionally, the retrospective cross-sectional design of this study is another limitation. Conducting future multicenter studies with a prospective design and using a larger sample size, along with assessment of clinical outcomes in cases with delayed IVI may provide more robust conclusions and help in developing comprehensive and realistic guidelines.

\section{CONCLUSIONS}

The number of IVIs and patients attending the retina clinic of a tertiary hospital decreased by more than 10-fold during the COVID-19 pandemic. These findings show that COVID-19 has turned the management of sightthreatening eye diseases into a challenging process. Ophthalmologists will have to face more complications in untreated patients, and will need to implement new ways of clinical practice using COVID-19 control measures, to protect both themselves and their patients from becoming infected.

\section{ETHICAL DECLARATIONS}

Ethical approval: The Ethics Committee of Ankara Training and Research Hospital approved our study, and written consent was obtained from all patients. This study adhered to the tenets of the Declaration of Helsinki. Conflict of interests: None

\section{FUNDING}

\section{None.}

\section{ACIKNOWLEGMENTS}

\section{None.}

\section{REFERENCES}

1. Kim LA, D’Amore PA. A Brief History of Anti-VEGF for the Treatment of Ocular Angiogenesis. Am J Pathol. 2012;181(2):376-9. doi: 10.1016/j.ajpath.2012.06.006 pmid: 22749677

2. Centers for Disease Control and Prevention (2021). 'People with Certain Medical Conditions, Updated May 13, 2021.' Available at: https://www.cdc.gov/coronavirus/2019-ncov/need-extra-precautions/people-with-medical-conditions.html (Accessed: May 14, 2021)

3. Gold MS, Sehayek D, Gabrielli S, Zhang X, McCusker C, Ben-Shoshan M. COVID-19 and comorbidities: a systematic review and meta-analysis. Postgrad Med. 2020;132(8):749-55. doi: 10.1080/00325481.2020.1786964 pmid: 32573311

4. American Society of Retina Specialists (2020). 'COVID-19: updates and resources'. Available at: https://www.asrs.org/practice/asrsmember-alert-regarding-covid-19-pandemic (Accessed: March 31, 2020)

5. Japanese Ophthalmological Society (2020). 'Guidance for ophthalmology-related healthcare workers regarding COVID-19'. Available at: https://www.nichigan.or.jp/tabid37.html?Search=Guidance+for+ophthalmology-related+healthcare+workers+regarding+COVID-19 (Accessed: March 31, 2020)

6. The Royal College of Ophthalmologists (2020). 'Medical retinal management plans during COVID-19'. Available at: https://www. rcophth.ac.uk/wp-content/uploads/2020/03/Medical-Retinal-Management-Plan-during-COVID-19-UPDATED-300320-1-3.pdf (Accessed: April 2, 2020)

7. Carnevali A, Giannaccare G, Gatti V, Scuteri G, Randazzo G, Scorcia V. Intravitreal injections during COVID-19 outbreak: Real-world experience from an Italian tertiary referral center. Eur J Ophthalmol. 2020;31(1):10-2. doi: 10.1177/1120672120962032 
pmid: 32967465

8. Korobelnik J-F, Loewenstein A, Eldem B, Joussen AM, Koh A, Lambrou GN, et al. Anti-VEGF intravitreal injections in the era of COVID-19: responding to different levels of epidemic pressure. Graefes Arch Clin Exp Ophthalmol. 2021;259(3):567-74. doi: 10.1007/ s00417-021-05097-0 pmid: 33528647

9. Korobelnik J-F, Loewenstein A, Vision Academy. Communicating with patients requiring anti-VEGF intravitreal injections and their families during the COVID-19 pandemic: an update. Graefes Arch Clin Exp Ophthalmol. 2021;259(3):795-7. doi: 10.1007/s00417020-05042-7 pmid: 33415358

10. TR Ministry of Health (2020). 'COVID-19 Information Platform'. Available at: https://covid19.saglik.gov.tr/TR-66300/covid-19nedir-.html (Accessed: April 2, 2020)

11. World Health Organization (WHO). 'Rational use of personal protective equipment for coronavirus disease (COVID-19) and considerations during severe shortages'. Available at: https://apps.who.int/iris/bitstream/handle/10665/331695/WHO-2019-nCovIPC_PPE_use-2020.3-eng.pdf (Accessed: April 2, 2020)

12. Olivia Li J-P, Shantha J, Wong TY, Wong EY, Mehta J, Lin H, et al. Preparedness among Ophthalmologists: During and Beyond the COVID-19 Pandemic. Ophthalmology. 2020;127(5):569-72. doi: 10.1016/j.ophtha.2020.03.037 Erratum in: Ophthalmology. 2020 Aug;127(8):1131. pmid: 32327128

13. Asadi S, Bouvier N, Wexler AS, Ristenpart WD. The coronavirus pandemic and aerosols: Does COVID-19 transmit via expiratory particles? Aerosol Sci Technol. 2020;54(6):635-8. doi: 10.1080/02786826.2020.1749229 pmid: 32308568

14. Wilson P, Zumla A. Transmission and prevention of acute viral respiratory tract infections in hospitals. Curr Opin Pulm Med. 2019;25(3):220-4. doi: 10.1097/MCP.0000000000000566 pmid: 30730312

15. Chandra S, Flanagan D, Hingorani M, Lotery A, Sivaprasad S. COVID19 and ophthalmology: a brief summary of the literature. Eye. 2020;34(7):1200-2. doi: 10.1038/s41433-020-0956-3 pmid: 32398838

16. Lau PE, Jenkins KS, Layton CJ. Current Evidence for the Prevention of Endophthalmitis in Anti-VEGF Intravitreal Injections. J Ophthalmol. 2018;2018:1-8. doi: 10.1155/2018/8567912 pmid: 30174946

17. Shmueli O, Chowers I, Levy J. Current safety preferences for intravitreal injection during COVID-19 pandemic. Eye. 2020;34(7):1165-7. doi: 10.1038/s41433-020-0925-x pmid: 32366999

18. Huemer J, Hienert J, Hirn C, Hackl C, Radda SM, Findl O. Remodelling intravitreal therapy pathways for macular disease during the COVID-19 pandemic and an Austrian national lockdown. BMJ Open Ophthalmol. 2020;5(1):e000560. doi: 10.1136/bmjophth-2020-000560 pmid: 34192151

19. Korobelnik J-F, Loewenstein A, Eldem B, Joussen AM, Koh A, Lambrou GN, et al. Guidance for anti-VEGF intravitreal injections during the COVID-19 pandemic. Graefes Arch Clin Exp Ophthalmol. 2020;258(6):1149-56. doi: 10.1007/s00417-020-04703-x pmid: 32328757

20. Saleh OA, Jammal H, Alqudah N, Alqudah A, Abu-Yaghi N. Clinical Experience in the Administration of Intravitreal Injection Therapy at a Tertiary University Hospital in Jordan During the COVID-19 Lockdown. Clin Ophthalmol. 2020;Volume 14:2473-80. doi: 10.2147/OPTH.S269179 pmid: 32943831

21. Romano F, Monteduro D, Airaldi M, Zicarelli F, Parrulli S, Cozzi M, et al. Increased Number of Submacular Hemorrhages as a Consequence of Coronavirus Disease 2019 Lockdown. Ophthalmol Retina. 2020;4(12):1209-10. doi: 10.1016/j.oret.2020.06.027 pmid: 32593777

22. Naravane AV, Mundae R, Zhou Y, Santilli C, van Kuijk FJGM, Nazari H, et al. Short term visual and structural outcomes of anti-vascular endothelial growth factor (anti-VEGF) treatment delay during the first COVID-19 wave: A pilot study. PloS One. 2021;16(2):e0247161. doi: 10.1371/journal.pone.0247161 pmid: 33596257

23. Yeter DY, Dursun D, Bozali E, Ozec AV, Erdogan H. Effects of the COVID-19 pandemic on neovascular age-related macular degeneration and response to delayed Anti-VEGF treatment. J Fr Ophtalmol. 2021;44(3):299-306. doi: 10.1016/j.jfo.2021.02.001 pmid: 33608176

24. Sevik MO, Aykut A, Özkan G, Dericioğlu V, Şahin Ö. The effect of COVID-19 pandemic restrictions on neovascular AMD patients treated with treat-and-extend protocol. Int Ophthalmol. 2021;41(9):2951-61. doi: 10.1007/s10792-021-01854-6 pmid: 33864577

25. The Royal College of Ophthalmologists (2020). 'Medical retinal management plans during COVID-19'. Available at: https://www. rcophth.ac.uk/wp-content/uploads/2020/10/Medical-Retinal-Management-Plan-During-COVID-19.pdf (Accessed: October 2, 2020)

26. Ashkenazy N, Goduni L, Smiddy WE. Short-Term Effects of COVID-19-Related Deferral of Intravitreal Injection Visits. Clin Ophthalmol. 2021;15:413-7. doi: 10.2147/OPTH.S296345 pmid: 33568895

27. Sophie R, Akhtar A, Sepah YJ, Ibrahim M, Bittencourt M, Do DV, et al. Aflibercept: a Potent Vascular Endothelial Growth Factor Antagonist for Neovascular Age-Related Macular Degeneration and Other Retinal Vascular Diseases. Biol Ther. 2012;2(1):3. doi: 10.1007/s13554-012-0003-4 pmid: 24392297 